\title{
Bibliometric Mapping of the Computational Intelligence Field
}

\author{
Nees Jan van Eck and Ludo Waltman
}

\begin{tabular}{|l|l|}
\hline \multicolumn{2}{|l|}{ ERIM REPORT SERIES RESEARCH IN MANAGEMENT } \\
\hline ERIM Report Series reference number & ERS-2007-027-LIS \\
\hline Publication & April 2007 \\
\hline Number of pages & 26 \\
\hline Persistent paper URL & http://hdl.handle.net/1765/10073 \\
\hline Email address corresponding author & nvaneck@few.eur.nl \\
\hline Address & Erasmus Research Institute of Management (ERIM) \\
& RSM Erasmus University / Erasmus School of Economics \\
& Erasmus Universiteit Rotterdam \\
& P.O.Box 1738 \\
& 3000 DR Rotterdam, The Netherlands \\
& Phone: + 31104081182 \\
& Fax: $\quad+31104089640$ \\
& Email: infoerim.eur.nl \\
& Internet: $\quad$ www.erim.eur.nl \\
\hline
\end{tabular}

Bibliographic data and classifications of all the ERIM reports are also available on the ERIM website: www.erim.eur.nl 


\section{ERASMUS RESEARCH INSTITUTE OF MANAGEMENT}

\section{REPORT SERIES}

\section{RESEARCH IN MANAGEMENT}

\begin{tabular}{|l|l|}
\hline ABSTRACT AND KEYWORDS \\
\hline Abstract & $\begin{array}{l}\text { In this paper, a bibliometric study of the computational intelligence field is presented. Bibliometric } \\
\text { maps showing the associations between the main concepts in the field are provided for the } \\
\text { periods 1996-2000 and 2001-2005. Both the current structure of the field and the evolution of } \\
\text { the field over the last decade are analyzed. In addition, a number of emerging areas in the field } \\
\text { are identified. It turns out that computational intelligence can best be seen as a field that is } \\
\text { structured around four important types of problems, namely control problems, classification } \\
\text { problems, regression problems, and optimization problems. Within the computational intelligence } \\
\text { field, the neural networks and fuzzy systems subfields are fairly intertwined, whereas the } \\
\text { evolutionary computation subfield has a relatively independent position. }\end{array}$ \\
\hline Free Keywords & $\begin{array}{l}\text { Bibliometrics, Bibliometric mapping, Computational intelligence, Neural networks, } \\
\text { Fuzzy systems, Evolutionary computation }\end{array}$ \\
\hline Availability & $\begin{array}{l}\text { The ERIM Report Series is distributed through the following platforms: } \\
\text { Academic Repository at Erasmus University (DEAR), DEAR ERIM Series Portal } \\
\text { Social Science Research Network (SSRN), SSRN ERIM Series Webpage } \\
\text { Research Papers in Economics (REPEC), REPEC ERIM Series Webpage }\end{array}$ \\
\hline Classifications & $\begin{array}{l}\text { The electronic versions of the papers in the ERIM report Series contain bibliographic metadata } \\
\text { by the following classification systems: } \\
\text { Library of Congress Classification, (LCC) } \underline{\text { LCC Webpage }} \\
\text { Journal of Economic Literature, (JEL), JEL Webpage } \\
\text { ACM Computing Classification System CCS Webpage } \\
\text { Inspec Classification scheme (ICS), ICS Webpage }\end{array}$ \\
\hline
\end{tabular}




\title{
Bibliometric Mapping \\ of the Computational Intelligence Field
}

\author{
Nees Jan van Eck \\ Ludo Waltman \\ Econometric Institute, Erasmus School of Economics \\ Erasmus University Rotterdam \\ P.O. Box 1738, 3000 DR Rotterdam, The Netherlands \\ E-mail: \{nvaneck,lwaltman\}@few.eur.nl
}

\begin{abstract}
In this paper, a bibliometric study of the computational intelligence field is presented. Bibliometric maps showing the associations between the main concepts in the field are provided for the periods 1996-2000 and 2001-2005. Both the current structure of the field and the evolution of the field over the last decade are analyzed. In addition, a number of emerging areas in the field are identified. It turns out that computational intelligence can best be seen as a field that is structured around four important types of problems, namely control problems, classification problems, regression problems, and optimization problems. Within the computational intelligence field, the neural networks and fuzzy systems subfields are fairly intertwined, whereas the evolutionary computation subfield has a relatively independent position.
\end{abstract}

\section{Keywords}

Bibliometrics, bibliometric mapping, computational intelligence, neural networks, fuzzy systems, evolutionary computation.

\section{Introduction}

In this paper, a bibliometric study of the field of computational intelligence $(\mathrm{CI})$ is presented. The CI field is analyzed by means of bibliometric maps that show the associations between the 
main concepts in the field. The maps provide insight into the structure of the CI field. More specifically, they visualize the division of the field into several subfields, and they indicate the relations between these subfields. By comparing bibliometric maps based on different periods of time, some insights are obtained into the evolution of the field over the last decade. The way in which the field has evolved is also studied through a quantitative analysis of the number of times researchers use specific concepts in their papers.

Bibliometric studies of the CI field are scarce. We are only aware of two studies in which the neural networks subfield is analyzed $[1,2]$. However, these studies are rather outdated, since they are based on data from the 1980s and the beginning of the 1990s. The present study is an extension of our earlier research [3,4], in which we analyzed the CI field based on papers presented at the IEEE World Congress on Computational Intelligence in 2002 and 2006. In the present study, we use data from three major journals and three major conferences over the period 1996-2005. By considerably increasing the amount of data on which our analysis is based, we expect to improve the reliability of our results compared to our earlier research. In the present study, we also discuss a method for assessing the stability of a bibliometric map. In our opinion, the stability of bibliometric maps usually does not get sufficient attention in bibliometric studies. By taking into account the stability of a map, the reliability of a bibliometric analysis can be improved significantly. A third improvement over our earlier research is the refinement of our methodology for constructing so-called concept density maps. The refined methodology better visualizes the amount of attention researchers pay to the various research topics in a field of science.

Bibliometric maps can be constructed in many different ways. Overviews of various approaches to bibliometric mapping are provided by Börner, Chen, and Boyack [5] and by Noyons [6]. The closely related field of information visualization is covered by Chen [7]. In this paper, we are concerned with maps in which the distance between two objects indicates the strength of the association between the objects. Objects that are located close to each other are regarded as strongly associated, whereas objects that are located far from each other are regarded as weakly associated or as not associated at all. In the field of bibliometrics, a number of approaches have been proposed for constructing this type of map. Most of these approaches rely on the method of multidimensional scaling [8]. The most popular approach seems to be the one that is discussed by McCain [9]. A good example of the application of this approach is provided by 
White and McCain [10]. In the present paper, we use our own approach to constructing bibliometric maps. Rather than on multidimensional scaling, our approach relies on a closely related method called VOS, which is an abbreviation for visualization of similarities. In our experience, our approach to constructing bibliometric maps provides better results than the approaches that have been proposed in the bibliometric literature. The focus of the present paper, however, is not on the methodological aspect of our research. Although we do provide a detailed description of our approach to constructing bibliometric maps, we do not discuss the differences with and the advantages over alternative approaches.

The paper is organized as follows. Our methodology for constructing bibliometric maps is discussed in Section 2. The bibliometric analysis of the CI field is presented in Section 3. Conclusions are drawn in Section 4.

\section{Methodology}

According to Börner et al. [5], the process of constructing a bibliometric map can be divided into the following six steps: (1) collection of raw data, (2) selection of the type of item to analyze, (3) extraction of relevant information from the raw data, (4) calculation of similarities between items based on the extracted information, (5) positioning of items in a low-dimensional space based on the similarities, and (6) visualization of the low-dimensional space. We now discuss the way in which we implement each of these steps in this paper. Our approach is summarized in Table 1.

The first step in the process of bibliometric mapping is the collection of raw data. In this paper, the raw data consist of a corpus containing abstracts of papers from three major journals and three major conferences in the CI field. ${ }^{1}$ The journals are the IEEE Transactions on Neural Networks, the IEEE Transactions on Fuzzy Systems, and the IEEE Transactions on Evolutionary Computation. The conferences are the International Joint Conference on Neural Networks, the IEEE International Conference on Fuzzy Systems, and the IEEE Congress on Evolutionary Computation. Both the journals and the proceedings of the conferences are published by the IEEE Computational Intelligence Society. Two sets of data are collected, one containing ab-

\footnotetext{
${ }^{1}$ Actually, the corpus not only contains abstracts of papers, it also contains titles. Both abstracts and titles are used to construct bibliometric maps. However, for simplicity we will only refer to the abstracts in the rest of this paper.
} 
Table 1: Summary of our implementation of the process of bibliometric mapping.

Step of the mapping process Implementation

(1) Collection of data

(2) Selection of type of item

(3) Extraction of information

(4) Calculation of similarities

(5) Positioning of items

(6) Visualization
Abstracts of papers from journals and

conferences in the CI field

Concepts

Co-occurrence frequency (Paragraph 2.1)

Association strength (Paragraph 2.2)

VOS (Paragraph 2.3)

Concept map (Paragraph 2.4)

Concept density map (Paragraph 2.5)

stracts from the period 1996-2000 and one containing abstracts from the period 2001-2005. In this way, separate bibliometric maps can be constructed for each of the two periods. The data are collected using two databases, IEEE Xplore and Elsevier Scopus. The latter database can be seen as an alternative to the well-known ISI Web of Science database. Compared to Web of Science, Scopus has the advantage that it also includes conference proceedings.

The second step in the process of bibliometric mapping is the selection of the type of item to analyze. According to Börner et al. [5], journals, papers, authors, and descriptive terms or words are most commonly selected as the type of item to analyze. Each type of item provides a different visualization of a field of science and results in a different analysis. In the present study, we choose to analyze concepts. ${ }^{2}$ A bibliometric map showing the associations between concepts in a scientific field is referred to as a concept map in this paper. To avoid any possible confusion, we note that our concept maps are very different from the concept maps originally introduced by Joseph D. Novak [11].

The third step in the process of bibliometric mapping is the extraction of relevant informa-

\footnotetext{
${ }^{2}$ According to the Merriam-Webster Online Dictionary, a concept is an abstract or generic idea generalized from particular instances. Concepts can be designated using terms. For example, the terms neural network, fuzzy system, and genetic algorithm designate three well-known concepts in the CI field. There may exist multiple terms designating the same concept. The terms neural network and neural net, for example, designate the same concept, and so do the terms fuzzy system, fuzzy inference system, and fuzzy logic system. Terms that designate the same concept are referred to as synonyms. In the case of synonyms, we have chosen a preferred term that we use to designate the corresponding concept in a consistent way throughout this paper.
} 
tion from the raw data collected in the first step. In this paper, the relevant information consists of the co-occurrence frequencies of concepts. The co-occurrence frequency of two concepts is extracted from a corpus of abstracts by counting the number of abstracts in which the two concepts both occur. To identify the concepts that occur in an abstract, one needs a thesaurus of the scientific field with which one is concerned. Because a thesaurus of the CI field is not available to us, we construct one ourselves. The approach that we take to construct a thesaurus of the CI field is discussed in Paragraph 2.1. We note that in the present study we do not use the same thesaurus as in our earlier research $[3,4]$. This is because the present study covers a longer period of time and, as a consequence, the concepts of interest may differ from our earlier research.

The fourth step in the process of bibliometric mapping is the calculation of similarities between items based on the information extracted in the third step. In this paper, similarities between items are calculated based on co-occurrence frequencies. In the bibliometric literature, two approaches can be distinguished for calculating similarities between items based on cooccurrence frequencies. One approach, which seems the most popular, is to use the Pearson correlation between the vectors of co-occurrence frequencies of two items as a measure of the items' similarity $[9,10]$. The other approach is to normalize co-occurrence frequencies using, for example, the cosine measure, the inclusion index, or the Jaccard index [12]. In this paper, we take the latter approach, since that approach is recommended in the statistical literature [8]. To normalize co-occurrence frequencies, we use a measure that we call association strength. A discussion of this measure is provided in Paragraph 2.2.

The fifth step in the process of bibliometric mapping is the positioning of items in a lowdimensional space based on the similarities calculated in the fourth step. In this paper, the low-dimensional space is referred to as a concept map and only two-dimensional concept maps are considered. In many studies $[9,10,12,13]$, the fifth step in the process of bibliometric mapping is performed using the method of multidimensional scaling [8]. However, it is our experience that multidimensional scaling does not always provide satisfactory results when it is used for bibliometric mapping. More specifically, when a large proportion of the similarities equal zero, which occurs quite frequently in bibliometric mapping, multidimensional scaling always provides maps in which the items lie more or less equally distributed within a circle (in the case of a two-dimensional map). To avoid this problem, we use a method that is closely related 
to multidimensional scaling. The method, which is called VOS, is discussed in Paragraph 2.3.

The sixth step in the process of bibliometric mapping is the visualization of the low-dimensional space that results from the fifth step. In our study, we use two different visualization approaches. We have implemented these approaches in two computer programs, which we call the concept map viewer and the concept density map viewer. The concept map viewer visualizes a concept map by displaying for each concept a label that indicates the location of the concept in the concept map. The concept density map viewer, on the other hand, displays labels only for a small number of frequently occurring concepts. In addition, this viewer uses colors to indicate the amount of attention researchers pay to the research topics located in the various areas of a concept map. The concept density map viewer is especially useful to get a quick overview of the division of a scientific field into several subfields and of the way in which subfields are related to each other. The visualizations provided by the concept map viewer and the concept density map viewer are discussed in more detail in Paragraph 2.4 and 2.5, respectively.

An issue that, in our opinion, usually does not get sufficient attention in bibliometric studies is the stability of bibliometric maps. Taking into account the issue of stability can significantly improve the reliability of a bibliometric analysis. We discuss a method for assessing the stability of a bibliometric map in Paragraph 2.6.

\subsection{Thesaurus}

To construct a thesaurus of the CI field, we make use of a term extraction tool that we have developed ourselves. The tool receives a corpus of abstracts as input. First, by using the MontyLingua software, ${ }^{3}$ the tool assigns a part-of-speech category (like verb, noun, or adjective) to each word in the corpus. Then, based on the assigned part-of-speech categories, the tool selects words or sequences of words that are likely to be terms. This is accomplished using a regular expression similar to the one proposed by Justeson and Katz [14]. The output of the tool is a list of candidate terms sorted by frequency of occurrence in the corpus. We manually validate the list of candidate terms. For each candidate term, we decide whether the term is relevant to the CI field. Furthermore, when we consider a candidate term relevant, we identify its synonyms. Synonymy relations are important because terms that are synonymous designate the same concept. The identification of synonyms is also done manually. Using the above procedure, we obtain

\footnotetext{
${ }^{3}$ See http://web.media.mit.edu/ hugo/montylingua/.
} 
a simple thesaurus of the CI field consisting of the field's most important terms as well as the synonymy relations between these terms. This thesaurus allows us to identify the concepts that occur in an abstract.

\subsection{Association strength}

To normalize co-occurrence frequencies of concepts, we use a measure that we call association strength. The aim of this measure is to normalize co-occurrence frequencies in such a way that concepts occurring in many abstracts and concepts occurring in only a few abstracts can be compared in a fair way. The association strength $a_{i j}$ of the concepts $i$ and $j$ is defined as

$$
a_{i j}=\frac{m c_{i j}}{c_{i i} c_{j j}} \quad \text { for } i \neq j,
$$

where $c_{i j}$ denotes the number of abstracts in which the concepts $i$ and $j$ both occur, $c_{i i}$ denotes the number of abstracts in which concept $i$ occurs, and $m$ denotes the total number of abstracts. The association strength of two concepts can be interpreted as the ratio between on the one hand the co-occurrence frequency of the concepts and on the other hand the expected co-occurrence frequency of the concepts obtained under the assumption that occurrences of the concepts are statistically independent [3]. To the best of our knowledge, there are, apart from our own research, only a few bibliometric studies in which the association strength measure is used $[12,13,15]$. In these studies, the measure is referred to as the proximity index. In our opinion, however, the association strength measure is preferable over alternative measures for normalizing co-occurrence frequencies, like the cosine measure, the inclusion index, and the Jaccard index. This is because the alternative measures do not always make fair comparisons between concepts with a high frequency of occurrence and concepts with a low frequency of occurrence.

\subsection{VOS}

The positioning of concepts in a concept map based on their association strengths is accomplished using a method that we call VOS, which is an abbreviation for visualization of similarities. We now briefly introduce this method. A more elaborate discussion of VOS, including an analysis of the relationship between VOS and multidimensional scaling, is provided elsewhere [16]. 
Let there be $n$ concepts. The aim of VOS is to provide a two-dimensional space in which the concepts $1, \ldots, n$ are located in such a way that the distance between any pair of concepts $i$ and $j$ reflects their association strength $a_{i j}$ as accurately as possible. Concepts that have a high association strength should be located close to each other, whereas concepts that have a low association strength should be located far from each other. The idea of VOS is to minimize a weighted sum of the squared Euclidean distances between all pairs of concepts. The higher the association strength of two concepts, the higher the weight of their squared distance in the summation. To avoid solutions in which all concepts are located at the same coordinates, the constraint is imposed that the sum of all distances must equal some positive constant. In mathematical notation, the objective function to be minimized in VOS is given by

$$
E\left(\mathbf{x}_{1}, \ldots, \mathbf{x}_{n}\right)=\sum_{i<j} a_{i j}\left\|\mathbf{x}_{i}-\mathbf{x}_{j}\right\|^{2}
$$

where the vector $\mathbf{x}_{i}=\left(x_{i 1}, x_{i 2}\right)$ denotes the location of concept $i$ in a two-dimensional space and $\|\cdot\|$ denotes the Euclidean norm. Minimization of the objective function is performed subject to the constraint

$$
\frac{1}{n(n-1)} \sum_{i<j}\left\|\mathbf{x}_{i}-\mathbf{x}_{j}\right\|=1
$$

Note that the distances $\left\|\mathbf{x}_{i}-\mathbf{x}_{j}\right\|$ in the constraint are not squared. We numerically solve the constrained optimization problem of minimizing (2) subject to (3) in two steps. We first convert the constrained optimization problem into an unconstrained optimization problem. We then solve the latter problem using a majorization algorithm [8]. To reduce the effect of local minima, we run the majorization algorithm using ten random starts. A computer program that implements the majorization algorithm is available online. ${ }^{4}$

\subsection{Concept map visualization}

To visualize a concept map, we use a Java applet that we call the concept map viewer. The concept map viewer indicates the location of a concept in a concept map by displaying a label at that location. This label shows a term that designates the concept. The viewer has scroll, zoom, and search functionality to support a comprehensive examination of a concept map. In addition to visualizing the associations between concepts, the viewer also visualizes the importance of concepts and the distribution of the interest in concepts over the neural networks,

\footnotetext{
${ }^{4}$ See www. nees janvaneck.nl/vos/.
} 
fuzzy systems, and evolutionary computation subfields. The importance of a concept, measured by counting the number of abstracts in which the concept occurs, is indicated by the size of the label representing the concept. The distribution of the interest in a concept over the neural networks, fuzzy systems, and evolutionary computation subfields, measured by calculating for each subfield the proportion of the abstracts in which the concept occurs, is indicated by the color of the label representing the concept. A color consists of a red, green, and blue component, each of which has a value between 0 and 255. Consider the color of the label representing concept $i$. The red, green, and blue component of this color are given by

$$
\begin{aligned}
& r\left(p_{i}^{\mathrm{FS}}, p_{i}^{\mathrm{NN}}, p_{i}^{\mathrm{EC}}\right)=\frac{p_{i}^{\mathrm{FS}}}{p_{i}^{\mathrm{FS}}+p_{i}^{\mathrm{NN}}+p_{i}^{\mathrm{EC}}} 180+75 \\
& g\left(p_{i}^{\mathrm{FS}}, p_{i}^{\mathrm{NN}}, p_{i}^{\mathrm{EC}}\right)=\frac{p_{i}^{\mathrm{NN}}}{p_{i}^{\mathrm{FS}}+p_{i}^{\mathrm{NN}}+p_{i}^{\mathrm{EC}}} 180+75
\end{aligned}
$$

and

$$
b\left(p_{i}^{\mathrm{FS}}, p_{i}^{\mathrm{NN}}, p_{i}^{\mathrm{EC}}\right)=\frac{p_{i}^{\mathrm{EC}}}{p_{i}^{\mathrm{FS}}+p_{i}^{\mathrm{NN}}+p_{i}^{\mathrm{EC}}} 180+75,
$$

respectively, where $p_{i}^{\mathrm{FS}}$ denotes the proportion of the abstracts from the IEEE Transactions on Fuzzy Systems and the IEEE International Conference on Fuzzy Systems in which concept $i$ occurs, $p_{i}^{\mathrm{NN}}$ denotes the proportion of the abstracts from the IEEE Transactions on Neural Networks and the International Joint Conference on Neural Networks in which concept $i$ occurs, and $p_{i}^{\mathrm{EC}}$ denotes the proportion of the abstracts from the IEEE Transactions on Evolutionary Computation and the IEEE Congress on Evolutionary Computation in which concept $i$ occurs. Using (4), (5), and (6), the color of a label is not influenced by differences in the number of papers published in the neural networks, fuzzy systems, and evolutionary computation subfields.

\subsection{Concept density map visualization}

A disadvantage of the concept map visualization discussed above is that labels of concepts usually overlap each other. This may obscure the overall structure of a concept map. Due to overlapping labels, it may for example be difficult to get a clear overview of the way in which a field of science is divided into subfields. To gain more insight into the overall structure of a concept map, we use a MATLAB program that we call the concept density map viewer. We refer to the maps shown by this viewer as concept density maps. Rather than displaying labels for all concepts, the concept density map viewer displays labels only for a small number 
of frequently occurring concepts. In addition, the viewer uses colors to indicate the amount of attention researchers pay to the research topics located in the various areas of a concept map. The amount of attention for a research topic is measured by counting the number of abstracts concerned with that topic. The idea of concept density maps has been introduced by van Eck, Frasincar, and van den Berg [17]. In this paragraph, we present a refinement of their methodology for constructing concept density maps.

Concept density maps are based on the notion of concept density. The concept density at a specific location in a concept map depends both on the number of neighboring concepts and on the importance of these concepts. The higher the number of neighboring concepts and the smaller the distance between these concepts and the location under consideration, the higher the concept density. Also, the more important the neighboring concepts, as indicated by the number of abstracts in which they occur, the higher the concept density. The general idea of a concept density map is that the amount of attention researchers pay to a research topic located in a specific area of a concept map is indicated by the concept density in that area. In a concept density map, colors are used to display the concept density in the various areas of a concept map. In this way, areas with a high concept density can be easily identified. Such areas contain concepts that together receive a lot of attention from researchers. Most likely, the areas therefore point to important research topics.

We now discuss the construction of concept density maps. The concept density at a specific location in a concept map is calculated by first placing a so-called kernel function at each concept location and then taking a weighted average of the kernel functions. The weight of a kernel function is set equal to the number of abstracts in which the corresponding concept occurs. In mathematical notation, the concept density at location $\mathbf{x}=\left(x_{1}, x_{2}\right)$ is given by

$$
D(\mathbf{x})=\frac{1}{h^{2} \sum_{i=1}^{n} c_{i i}} \sum_{i=1}^{n} c_{i i} K\left(\frac{x_{1}-x_{i 1}}{h}, \frac{x_{2}-x_{i 2}}{h}\right),
$$

where $K$ denotes a kernel function and $h$ denotes a smoothing parameter. Recall further that $c_{i i}$ denotes the number of abstracts in which concept $i$ occurs and $\mathbf{x}_{i}=\left(x_{i 1}, x_{i 2}\right)$ denotes the location of concept $i$ in a concept map. The kernel function $K$ must satisfy the conditions

$$
\begin{aligned}
& \forall t_{1}, t_{2}, t_{3}, t_{4}: t_{1}^{2}+t_{2}^{2}=t_{3}^{2}+t_{4}^{2} \Rightarrow K\left(t_{1}, t_{2}\right)=K\left(t_{3}, t_{4}\right), \\
& \forall t_{1}, t_{2}, t_{3}, t_{4}: t_{1}^{2}+t_{2}^{2}<t_{3}^{2}+t_{4}^{2} \Rightarrow K\left(t_{1}, t_{2}\right) \geq K\left(t_{3}, t_{4}\right),
\end{aligned}
$$


and

$$
\forall t_{1}, t_{2}: K\left(t_{1}, t_{2}\right) \geq 0
$$

A kernel function satisfying these conditions is invariant to rotation. We require this property because concept maps are also invariant to rotation. In this paper, we use the bivariate standard normal distribution for the kernel function $K$, which means that

$$
K\left(t_{1}, t_{2}\right)=\frac{1}{2 \pi} \exp \left(-\frac{t_{1}^{2}+t_{2}^{2}}{2}\right) .
$$

The smoothness of the concept density function in (7) is determined by the smoothing parameter $h$. Choosing an appropriate value for $h$ is essential. A too small value for $h$ results in a concept density function that is too rough, whereas a too large value results in a concept density function that is too smooth. The coloring of a concept density map is based on concept densities calculated using (7). We use colors ranging from blue to red in our research. Blue areas in a concept density map have the lowest concept density and thus point to research topics that receive very little attention from researchers. Red areas, on the other hand, have the highest concept density and thus point to research topics that receive a lot of attention from researchers.

As a final remark, we note that the above approach to calculating concept densities is mathematically somewhat similar to the statistical technique of kernel density estimation. This technique is discussed by, for example, Scott [18].

\subsection{Stability}

A bibliometric map can be considered stable if small changes in the underlying data produce only small changes in the map [19]. Although the concept maps presented in this paper are constructed using VOS, the stability of the maps can be analyzed in a similar way as in the case of maps constructed using multidimensional scaling methods. De Leeuw and Meulman [19] propose to analyze the stability of multidimensional scaling maps by studying the effect of leaving out one object. Other approaches to stability analysis, proposed by Heiser and Meulman [20, 21] and Weinberg, Carroll, and Cohen [22], investigate the effect of random sampling on multidimensional scaling maps. The latter approaches all rely on the statistical technique of bootstrapping.

Our analysis of the stability of our concept maps also focuses on the effect of random sampling. The approach that we take is quite similar to the one discussed by Heiser and Meulman 
[21]. When constructing a concept map, the corpus of abstracts on which the map is based can be regarded as a sample, with each abstract representing an observation. The sample defines an empirical probability distribution over abstracts. A bootstrap sample is a sample that is drawn, with replacement, from this empirical probability distribution. A bootstrap sample has the same size as the original sample. In this paper, 100 bootstrap samples are drawn in order to analyze the stability of a concept map. For each bootstrap sample, a concept map is constructed using the methodology discussed above. Since concept maps are invariant to rotation, reflection, translation, and dilation (i.e., stretching and shrinking), we cannot directly compare the concept maps obtained from the different bootstrap samples. Instead, we first use Procrustes rotation [8] to match each concept map as closely as possible to the concept map obtained from the original sample. In this way, we end up with 100 concept maps that can be used to analyze the stability of individual concepts. For each concept, we thus have 100 locations, each obtained from a different bootstrap sample. To analyze the stability of a concept in a concept map, we draw an ellipse that covers most of the bootstrap locations of the concept. The ellipse is centered at the average of the bootstrap locations. The shape of the ellipse is based on the assumption of a bivariate normal sampling distribution and depends on the standard deviations and the correlation estimated using the bootstrap procedure. The size of the ellipse is determined in such a way that the ellipse covers exactly $90 \%$ of the bootstrap locations. In this way, an ellipse can be interpreted as an approximate $90 \%$ confidence region for the location of a concept.

\section{Analysis}

As stated before, our analysis is based on abstracts of papers from three major journals and three major conferences in the CI field. Furthermore, two time periods are considered in the analysis, 1996-2000 and 2001-2005. For each period, the number of abstracts that we obtained from the different journals and conference proceedings is reported in Table $2 .{ }^{5}$ Based on the abstracts, we constructed a thesaurus of the CI field using the approach discussed in Paragraph 2.1. We ended up with a thesaurus containing 376 concepts. However, when constructing concept maps of the CI field, we only included concepts that occurred in at least ten abstracts. This was done because we considered the amount of data on concepts occurring in less than ten abstracts too limited

\footnotetext{
${ }^{5}$ Since the first issue of the IEEE Transactions on Evolutionary Computation appeared in 1997, abstracts from this journal were not available for the year 1996.
} 
Table 2: Number of abstracts in the corpus.

\begin{tabular}{lrr}
\hline Journal / conference proceedings & \multicolumn{2}{c}{ Number of abstracts } \\
& 1996-2000 & 2001-2005 \\
\hline \hline IEEE Trans. Neural Networks & 701 & 682 \\
IEEE Trans. Fuzzy Systems & 272 & 360 \\
IEEE Trans. Evolutionary Computation & 89 & 203 \\
Proc. Int. Joint Conf. Neural Networks & 2761 & 2761 \\
Proc. IEEE Int. Conf. Fuzzy Systems & 1452 & 1148 \\
Proc. IEEE Congr. Evolutionary Computation & 960 & 1629 \\
\cline { 2 - 2 } & 6235 & 6783 \\
\hline
\end{tabular}

for a reliable analysis. In the periods 1996-2000 and 2001-2005, there were, respectively, 332 and 337 concepts that occurred in at least ten abstracts. For these concepts, we counted the co-occurrence frequencies. In both periods, $74 \%$ of the co-occurrence frequencies turned out to be equal to zero, which indicates that most combinations of concepts did not occur in any abstract at all. The concept maps that we constructed for the periods 2001-2005 and 19962000 are shown in Figures 1 and 4, respectively. The corresponding concept density maps are shown in Figures 2 and 5. Since the figures are printed in black and white, the coloring of the labels (see Paragraph 2.4) is not visible in the concept maps. Similarly, in the concept density maps, colors indicating the density of concepts (see Paragraph 2.5) are not visible. Instead, curves that indicate points of equal density are shown in the concept density maps. Concept maps and concept density maps with the correct coloring are available online. ${ }^{6}$ We encourage the interested reader to have look at these maps, since they are much more insightful than maps printed in black and white. Moreover, we have also made available online our concept map viewer (see Paragraph 2.4). Using this viewer, the concept maps in Figures 1 and 4 can be examined in much more detail. To provide some insight into the stability of our concept maps, approximate $90 \%$ confidence regions for a number of frequently occurring concepts in the periods 2001-2005 and 1996-2000 are shown in Figures 3 and 6, respectively. The confidence regions were calculated using the bootstrap approach discussed in Paragraph 2.6.

\footnotetext{
${ }^{6}$ See www.neesjanvaneck.nl/ijufks/.
} 


\subsection{Structure of the computational intelligence field}

To analyze the current structure of the CI field, we consider the maps for the period 2001-2005, which are shown in Figures 1, 2, and 3. Our initial expectation was to find three well-separated clusters of concepts, corresponding to the three well-known subfields of the CI field, that is, neural networks, fuzzy systems, and evolutionary computation. This is also what we found in our earlier research [3, 4], in which we used a smaller data set and a smaller thesaurus than in the present study. However, somewhat to our surprise, there is no very clear correspondence between on the one hand the clusters that can be observed in our maps and on the other hand the three subfields of the CI field. The clusters can be seen most easily in the concept density map in Figure 2. The cluster in the right part of the map clearly corresponds to the evolutionary computation subfield, but the clusters in the left part of the map do not correspond one-to-one to the neural networks and fuzzy systems subfields. Instead, the clustering in the left part of the map seems to reflect different types of problems that are studied in the CI field. In the lower left part, there is a cluster for control problems. In the upper left part, there is a cluster for classification problems, that is, for problems involving the prediction of a class label. And in the center of the left part, there is a cluster for problems in which a continuous value has to be predicted. We will refer to the latter problems as regression problems. Moreover, the interpretation of clusters in terms of the type of problem with which they are concerned can also be applied to the cluster in the right part of the map. Since evolutionary computation primarily deals with optimization, this cluster can be seen as a cluster for optimization problems. So, following the above interpretation of the maps for the period 2001-2005, it turns out that, contrary to our expectation, the CI field is not structured around the three most important techniques studied in the field, that is, neural networks, fuzzy systems, and evolutionary computation. Instead, the field is structured around what seem to be the four main types of problems with which the field is concerned. These types of problems are control problems, classification problems, regression problems, and optimization problems.

A closer examination of the concept map for the period 2001-2005, either using Figure 1 or using the concept map viewer available online, reveals that each of the three clusters in the left part of the map contains both concepts from the neural networks subfield and concepts from the fuzzy systems subfield. The control cluster is dominated by fuzzy systems concepts, but the cluster also contains some neural networks concepts, for example recurrent neural network, 


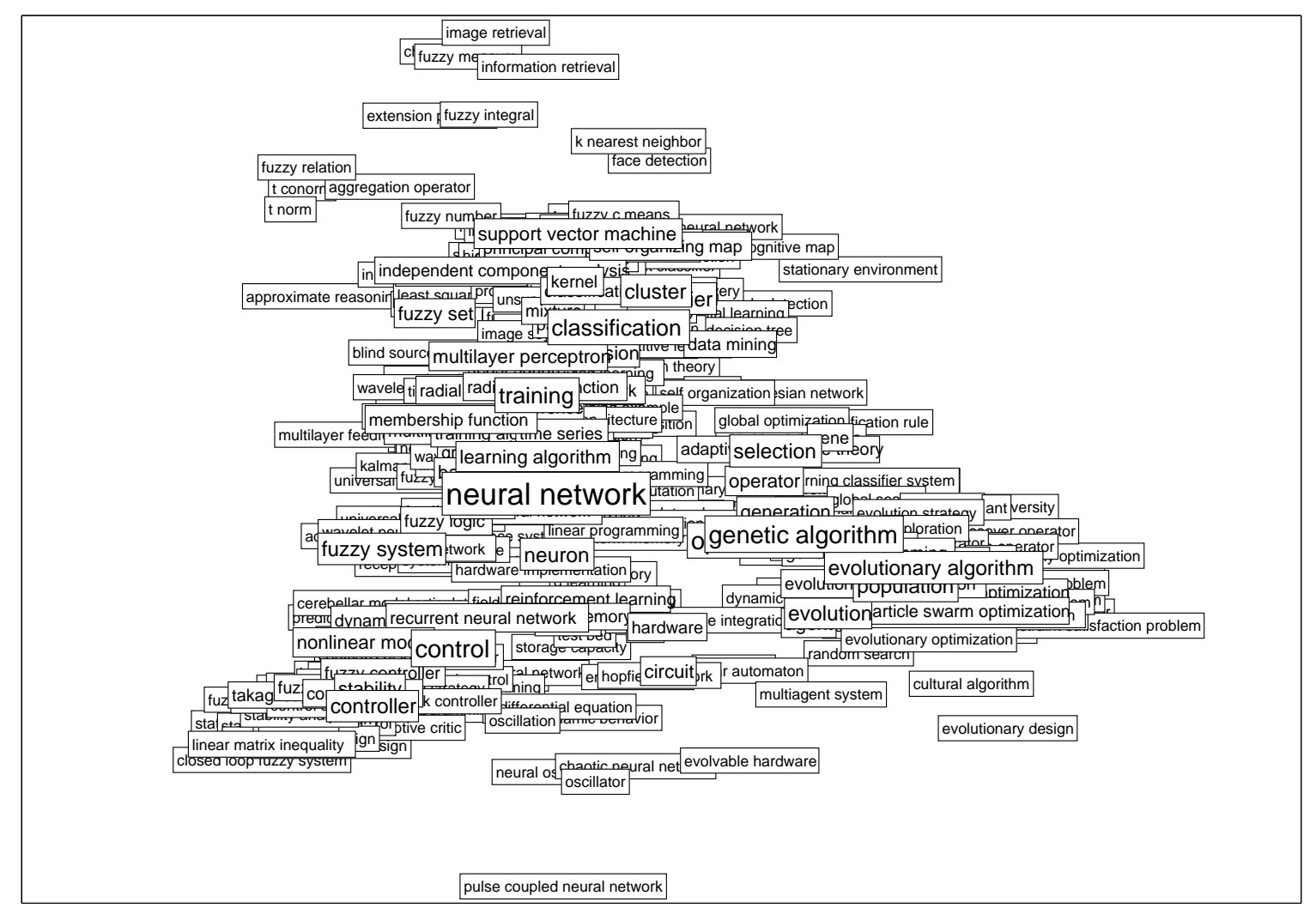

Figure 1: Concept map for the period 2001-2005.

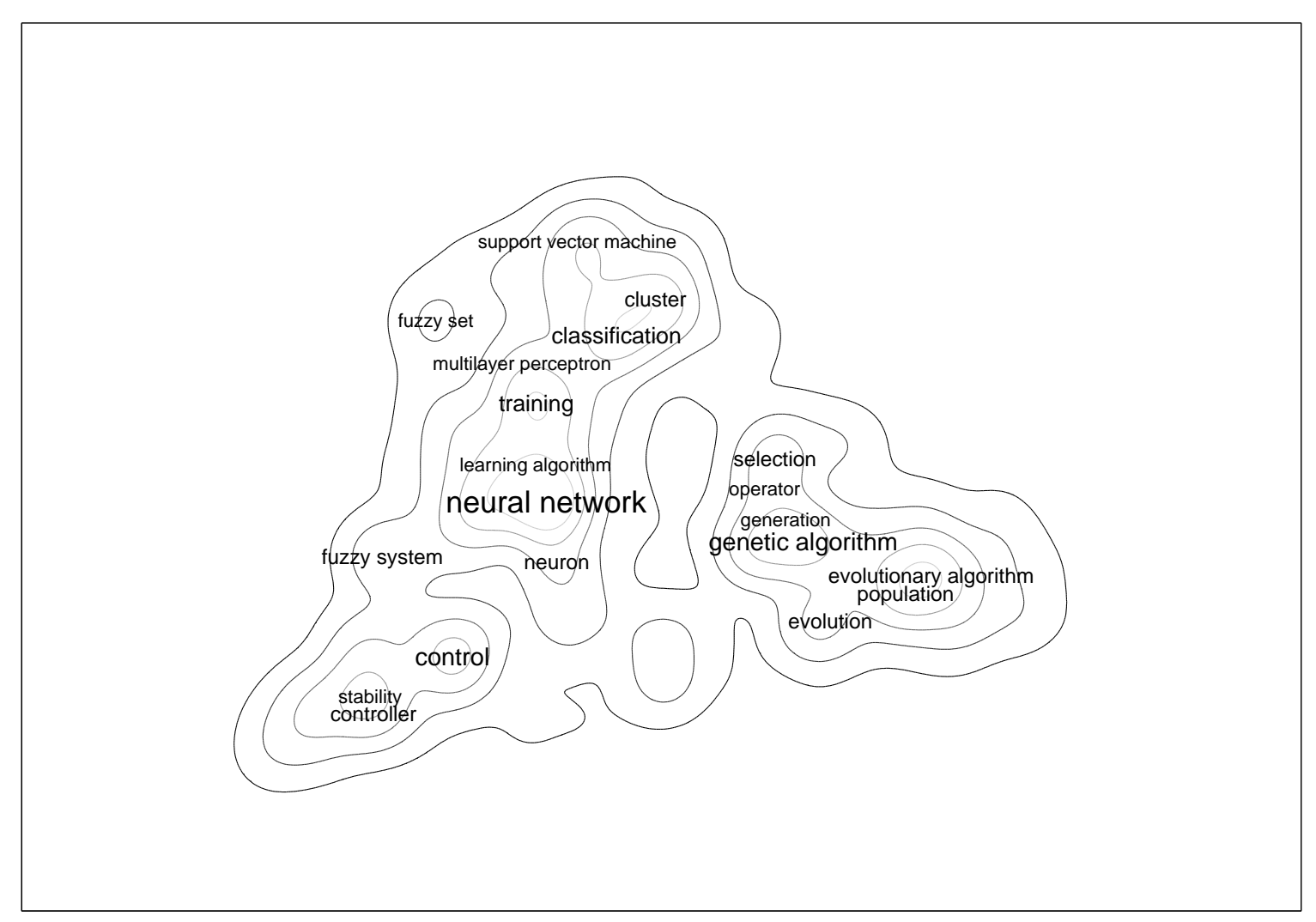

Figure 2: Concept density map for the period 2001-2005. 


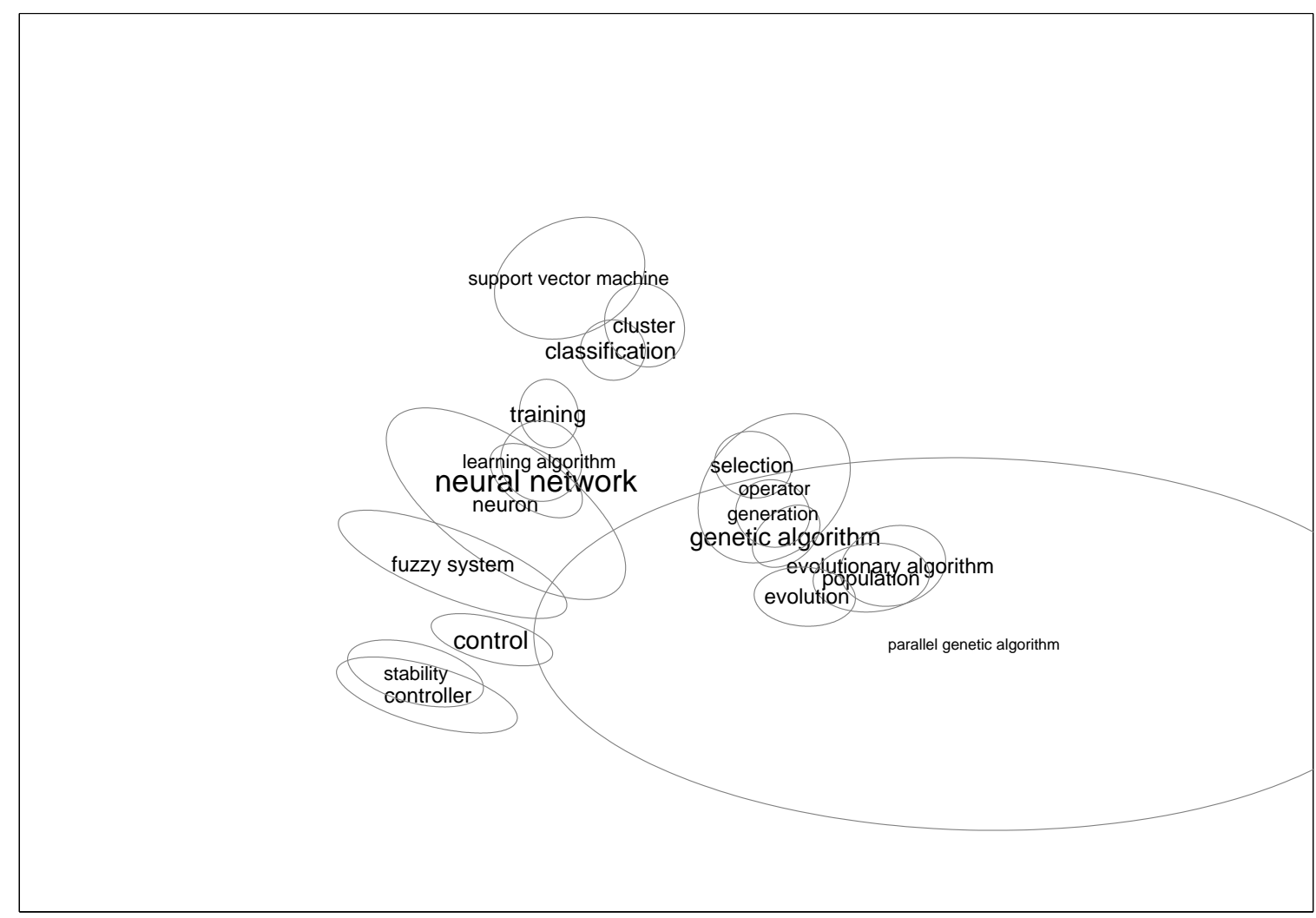

Figure 3: Approximate $90 \%$ confidence regions for a number of frequently occurring concepts in the period 2001-2005.

neural network controller, and neural system. Most concepts in the classification and regression clusters, on the other hand, belong to the neural networks subfield, but there are also a number of fuzzy systems concepts in these clusters. Some examples are fuzzy c-means, fuzzy clustering, and fuzzy classifier in the classification cluster and membership function, fuzzy inference, and defuzzification in the regression cluster. Together, all these examples clearly indicate that the clustering found in our maps does not coincide with the division of the CI field into the neural networks, fuzzy systems, and evolutionary computation subfields. More specifically, the neural networks and fuzzy systems subfields turn out to be fairly intertwined. The evolutionary computation subfield, on the other hand, has a relatively independent position within the CI field.

Based on the maps, some further observations on the structure of the CI field can be made. The concept density map in Figure 2 shows that the classification cluster and the regression cluster are only weakly separated from each other. The separation between other clusters is much stronger. One might even argue, based on the concept density map, that there is in fact one 
large cluster, which is concerned with both classification and regression problems. The weak separation between the classification cluster and the regression cluster seems to indicate that classification and regression problems are seen as fairly similar. This is probably due to the fact that important CI techniques like neural networks and fuzzy systems can be applied to both types of problems. Using the concept map, it can further be observed that within the classification cluster there is no clear separation between concepts related to classification (e.g., classification, support vector machine, and neural network classifier) on the one hand and concepts related to clustering (e.g., cluster, fuzzy c-means, and fuzzy clustering) on the other hand. Apparently, researchers do not see much difference between classification and clustering.

We now consider the map in Figure 3, which shows approximate 90\% confidence regions for a number of frequently occurring concepts in the period 2001-2005. It can be seen that some concepts, like neuron and fuzzy system, are quite unstable. Other concepts, like genetic algorithm and classification, are much more stable. For comparison, the concept parallel genetic algorithm, which occurs in only ten abstracts, is also shown in the map. This concept is highly unstable, as indicated by its very large confidence region. Although concepts with confidence regions of this size are rather exceptional, it turns out that, on average, less frequently occurring concepts are also less stable. This is because the locations of these concepts in a concept map are calculated from a relatively small amount of data. The example of parallel genetic algorithm shows that one should be very careful when making detailed statements based on the location of a single concept, especially if the concept occurs in only a few abstracts. The above analysis of the structure of the CI field does not contain any very detailed statement, and it therefore does not depend too strongly on the exact locations of individual concepts. In our opinion, a more detailed analysis may be possible, but such an analysis should be performed very carefully.

\subsection{Evolution of the computational intelligence field over the last decade}

To analyze the evolution of the CI field over the last decade, we first consider the differences in the number of occurrences of concepts in the periods 1996-2000 and 2001-2005. In Table 3, the concepts are listed that have the largest relative increase in their number of occurrences between the two periods. Only concepts occurring in at least 20 abstracts in the period 2001-2005 are shown. Similarly, the concepts with the largest relative decrease in their number of occurrences are listed in Table 4. This table only shows concepts that occur in at least 20 abstracts in the 
Table 3: Concepts with the largest relative increase in their number of occurrences.

\begin{tabular}{|c|c|c|}
\hline \multirow[t]{2}{*}{ Concept } & \multicolumn{2}{|c|}{ Number of occurrences } \\
\hline & 1996-2000 & $2001-2005$ \\
\hline genetic regulatory network & 0 & 26 \\
\hline NSGA-II & 0 & 22 \\
\hline least squares support vector machine & 1 & 27 \\
\hline artificial immune system & 2 & 34 \\
\hline evolutionary multiobjective optimization & 3 & 36 \\
\hline particle swarm optimization & 10 & 113 \\
\hline pareto front & 5 & 41 \\
\hline gaussian kernel & 3 & 21 \\
\hline ant colony optimization & 4 & 28 \\
\hline support vector machine & 39 & 264 \\
\hline multiobjective evolutionary algorithm & 11 & 70 \\
\hline learning classifier system & 4 & 25 \\
\hline support vector & 12 & 71 \\
\hline association rule & 5 & 23 \\
\hline long term memory & 5 & 21 \\
\hline pareto optimal solution & 6 & 24 \\
\hline ant & 14 & 51 \\
\hline immune system & 10 & 34 \\
\hline kernel & 54 & 173 \\
\hline multiobjective optimization & 35 & 112 \\
\hline differential evolution & 11 & 35 \\
\hline ant colony & 8 & 25 \\
\hline gene & 52 & 135 \\
\hline mutual information & 19 & 49 \\
\hline image retrieval & 11 & 27 \\
\hline
\end{tabular}

period 1996-2000. For each concept in Tables 3 and 4, the number of abstracts in which the concept occurs in the periods 1996-2000 and 2001-2005 is reported. 
Table 4: Concepts with the largest relative decrease in their number of occurrences.

\begin{tabular}{|c|c|c|}
\hline \multirow[t]{2}{*}{ Concept } & \multicolumn{2}{|c|}{ Number of occurrences } \\
\hline & 1996-2000 & $2001-2005$ \\
\hline fuzzy constraint & 21 & 4 \\
\hline constructive algorithm & 28 & 8 \\
\hline cascade correlation & 23 & 7 \\
\hline fuzzy logic control & 48 & 15 \\
\hline multilayer feedforward neural network & 44 & 16 \\
\hline control action & 33 & 13 \\
\hline hidden unit & 117 & 48 \\
\hline iris data & 31 & 13 \\
\hline fuzzy number & 63 & 27 \\
\hline evolutionary programming & 90 & 39 \\
\hline fuzzy control system & 73 & 32 \\
\hline feedforward neural network & 184 & 82 \\
\hline sliding mode controller & 20 & 9 \\
\hline universal approximator & 31 & 14 \\
\hline fuzzy logic controller & 128 & 58 \\
\hline defuzzification & 44 & 20 \\
\hline knowledge base & 78 & 37 \\
\hline PID controller & 41 & 20 \\
\hline rule extraction & 43 & 21 \\
\hline inverted pendulum & 57 & 28 \\
\hline expert system & 51 & 26 \\
\hline approximate reasoning & 25 & 13 \\
\hline backpropagation & 398 & 211 \\
\hline fuzzy controller design & 22 & 12 \\
\hline output layer & 42 & 23 \\
\hline
\end{tabular}

The data in Table 3 indicate a number of emerging areas in the CI field. Interestingly, most of these areas lie in the evolutionary computation subfield. The data reveal six emerging 
areas in this subfield. These areas are genetic regulatory networks, evolutionary multiobjective optimization, artificial immune systems, particle swarm optimization, ant colony optimization, and differential evolution. Furthermore, the interest of evolutionary computation researchers in the area of learning classifier systems has also increased considerably over the last years. As can be seen in Table 2, the recent developments in the evolutionary computation subfield have resulted in a large increase in the number of papers from this subfield. Another emerging area revealed by the data in Table 3 is support vector machines. Most abstracts containing the concept support vector machine belong to papers from the IEEE Transactions on Neural Networks or the International Joint Conference on Neural Networks. This shows that support vector machines research is usually seen as part of the neural networks subfield. Given the fairly large number of papers concerned with support vector machines, it is quite remarkable that the topic of support vector machines is not covered in two recent textbooks on CI [23, 24]. Apparently, there is no complete consensus within the CI community on the question whether support vector machines research belongs to the CI field at all. In the fuzzy systems subfield, research interest in the topic of fuzzy association rules has increased significantly over the last decade. This is indicated by the concept association rule in Table 3.

Obviously, there must also be areas with a decreasing interest of CI researchers. These areas are indicated by the data in Table 4. In the neural networks subfield, interest in the area of feedforward neural networks has decreased considerably. The same is true for the area of fuzzy control in the fuzzy systems subfield. In the evolutionary computation subfield, the amount of research in the area of evolutionary programming has clearly decreased.

We now compare the maps for the period 1996-2000, shown in Figures 4, 5, and 6, to the maps for the period 2001-2005, shown in Figures 1, 2, and 3. The concept density map in Figure 5 reveals that in the period 1996-2000 the CI field was largely structured around the three most important techniques studied in the field, that is, neural networks, fuzzy systems, and evolutionary computation. The map clearly shows three clusters, each corresponding to one of the three techniques. The correspondence between the three clusters and the three techniques is not perfect. By examining the concept map for the period 1996-2000, either using Figure 4 or using the concept map viewer available online, it can be seen that some fuzzy systems concepts are located in the neural networks cluster. Most of these concepts have to do with classification (e.g., fuzzy classifier and fuzzy classification), clustering (e.g., fuzzy clustering and fuzzy c-means), or 


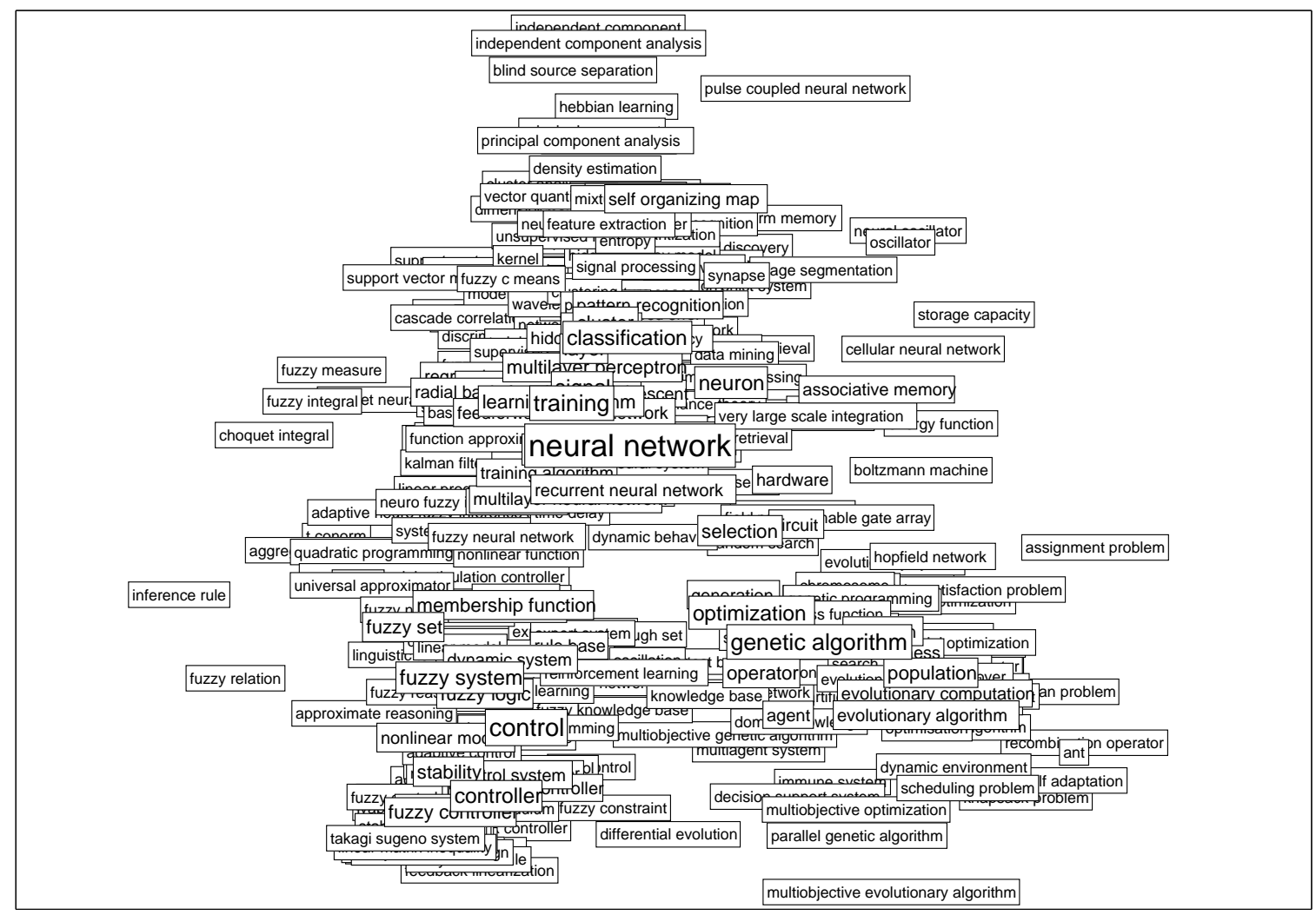

Figure 4: Concept map for the period 1996-2000.

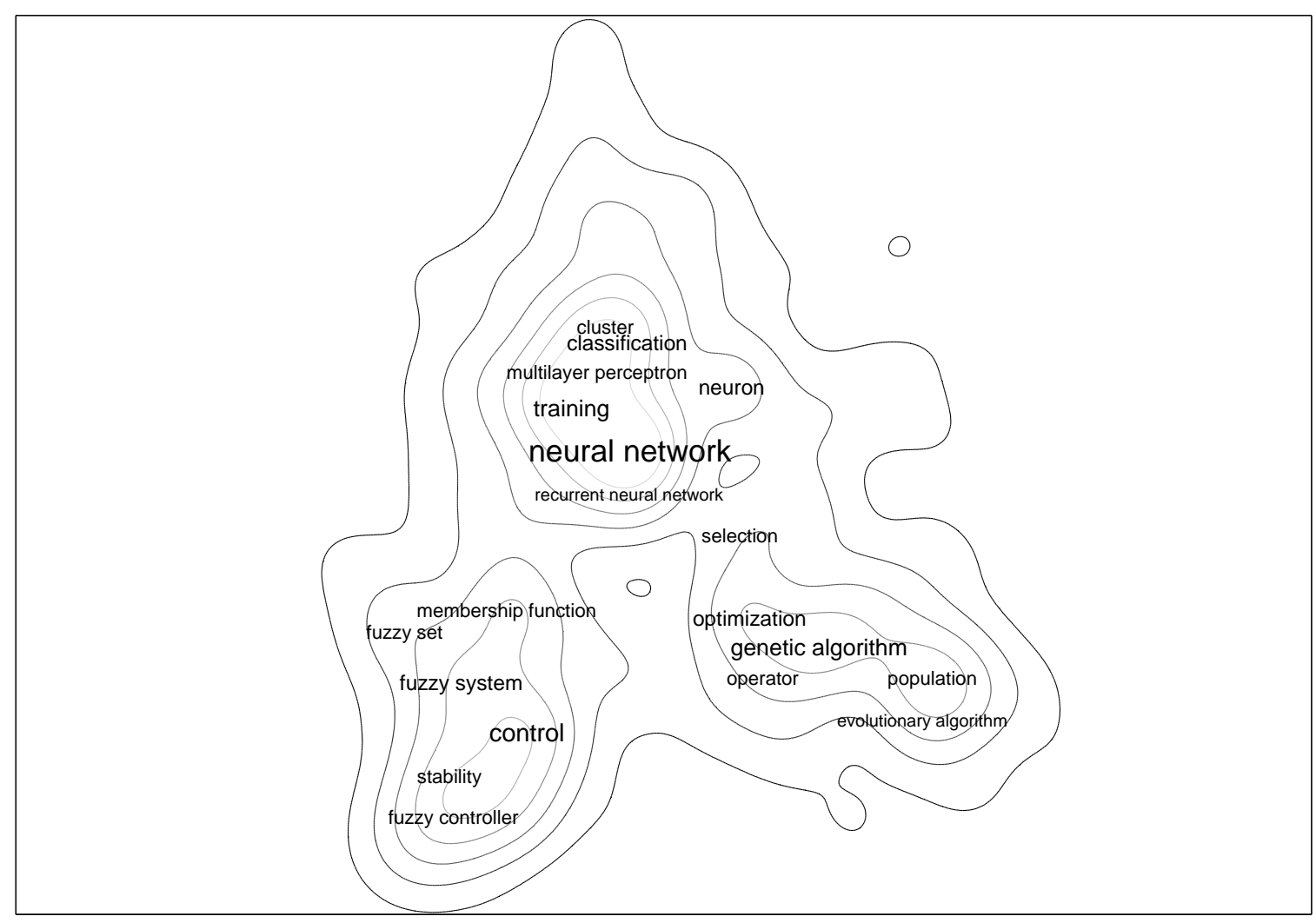

Figure 5: Concept density map for the period 1996-2000. 


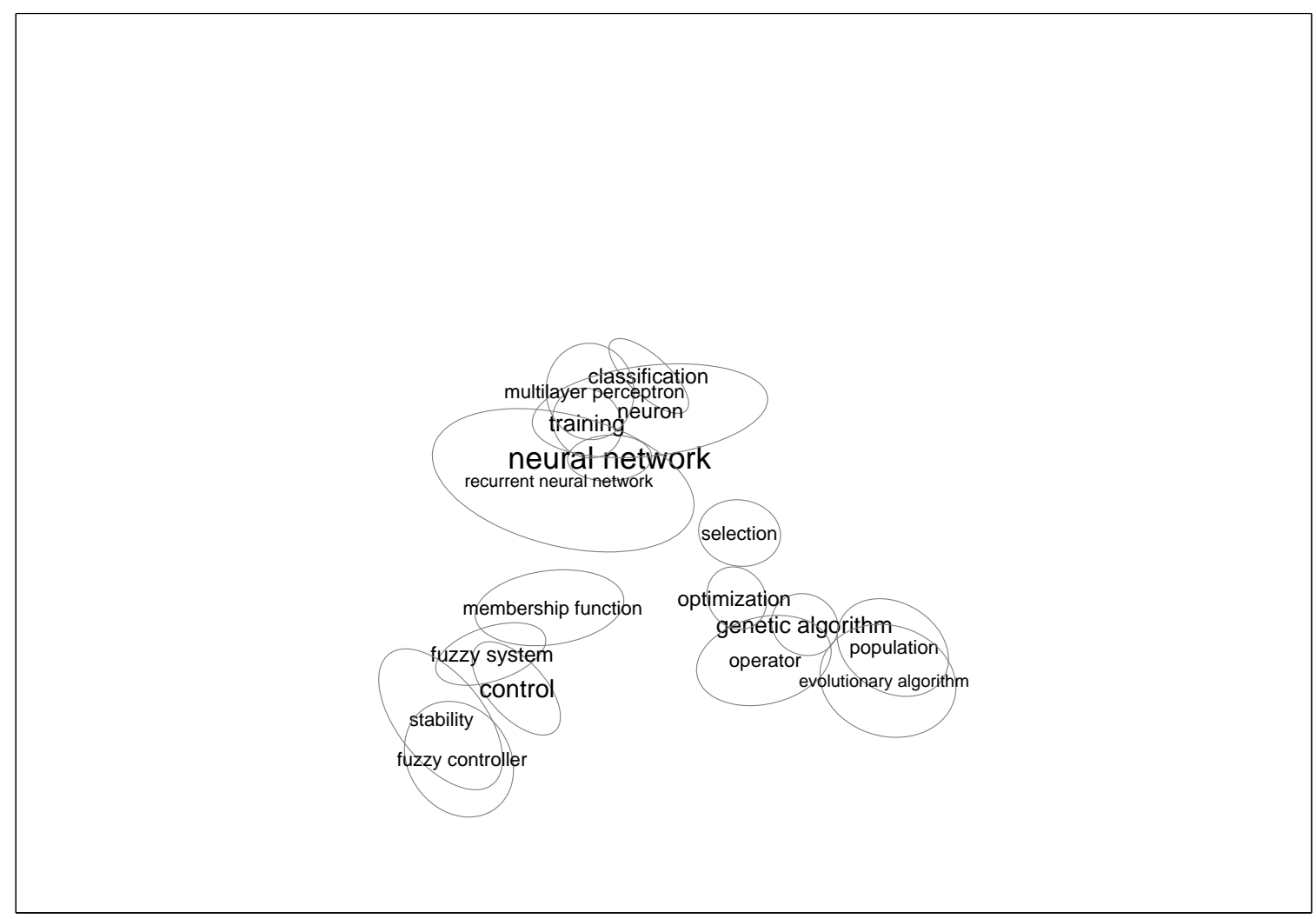

Figure 6: Approximate $90 \%$ confidence regions for a number of frequently occurring concepts in the period 1996-2000.

neuro-fuzzy systems (e.g., fuzzy neural network and neuro-fuzzy inference system). However, even though the correspondence between the three clusters and the three most important CI techniques is not perfect, it is clear that in the period 1996-2000 the CI field was much more structured around techniques than it was in the period 2001-2005. As discussed above, in the latter period the field was structured around four types of problems that each receive a lot of attention in the field.

Based on the concept density maps in Figures 2 and 5, some further observations on the evolution of the CI field can be made. One thing to note is that in the map for the period 1996-2000 concepts related to classification and concepts related to regression are located much closer to each other than in the map for the period 2001-2005. Apparently, nowadays research into classification problems on the one hand and into regression problems on the other hand is somewhat more separated than it was some years ago. Another observation is that concepts related to control and concepts related to neural networks have moved toward each other. This might be an indication that the application of neural network techniques to control problems 
has increased over the last decade.

\section{Conclusions}

In this paper, we have presented a bibliometric study of the CI field. Based on our analysis, we can draw a number of conclusions. First of all, our initial expectation that the CI field is structured around the neural networks, fuzzy systems, and evolutionary computation subfields turns out to be too simplistic. As revealed by our bibliometric maps for the period 2001-2005, the CI field can best be seen as a field that is structured around four important types of problems, namely control problems, classification problems, regression problems, and optimization problems. Moreover, the neural networks and fuzzy systems subfields turn out to be fairly intertwined. Both subfields are concerned with control, classification, and regression problems. The evolutionary computation subfield mainly deals with optimization problems, and it therefore turns out to have a relatively independent position within the CI field. Interestingly, the intertwining of the neural networks and fuzzy systems subfields has increased considerably over the last decade. This can be seen by comparing the maps for the period 2001-2005 to the maps for the period 1996-2000. In the latter maps, the neural networks and fuzzy systems subfields are clearly separated from each other. Apparently, in the last decade there must have been some development in the CI field that has brought the neural networks and fuzzy systems subfields closer together. A possible explanation might be that more and more researchers recognize that in many cases neural network techniques and fuzzy system techniques are applied to rather similar problems, even though the techniques themselves are very different. As a consequence, more and more researchers become interested in comparing the two types of techniques, and they start combining them into hybrid systems. So, researchers focus less on one type of technique. Instead, they focus on the problem with which they are concerned, and they try to find the technique or the combination of techniques that solves the problem in the most satisfactory way.

Our analysis of the frequency with which researchers use specific concepts in their papers has revealed a number of emerging areas in the CI field. These areas are genetic regulatory networks, evolutionary multiobjective optimization, artificial immune systems, particle swarm optimization, ant colony optimization, differential evolution, and support vector machines. In- 
terestingly, most of these areas lie in the evolutionary computation subfield, which suggests that this subfield has been particularly innovative over the last decade. We also note that it is not completely clear whether the area of support vector machines should be seen as part of the CI field at all. The interest of CI researchers in a number of more traditional research topics has decreased significantly over the last decade. These topics are feedforward neural networks, fuzzy control, and evolutionary programming.

\section{Acknowledgement}

We would like to thank Joost van Rosmalen for his helpful suggestions concerning the stability analysis of bibliometric maps.

\section{References}

[1] A. F. J. van Raan and R. J. W. Tijssen. The neural net of neural network research: an exercise in bibliometric mapping. Scientometrics, 26(1):169-192, 1993.

[2] E. C. M. Noyons and A. F. J. van Raan. Monitoring scientific developments from a dynamic perspective: self-organized structuring to map neural network research. J. Am. Soc. Inf. Sci. Tec., 49(1):68-81, 1998.

[3] N. J. van Eck, L. Waltman, J. van den Berg, and U. Kaymak. Visualizing the WCCI 2006 knowledge domain. In Proc. 2006 IEEE Int. Conf. Fuzzy Systems, pages 7862-7869, 2006.

[4] N. J. van Eck, L. Waltman, J. van den Berg, and U. Kaymak. Visualizing the computational intelligence field. IEEE Computational Intelligence Magazine, 1(4):6-10, 2006.

[5] K. Börner, C. Chen, and K. W. Boyack. Visualizing knowledge domains. Annu. Rev. Inform. Sci., 37:179-255, 2003.

[6] E. C. M. Noyons. Science maps within a science policy context. In H. F. Moed, W. Glänzel, and U. Schmoch, editors, Handbook of Quantitative Science and Technology Research, pages 237-255. Kluwer Academic Publishers, 2004.

[7] C. Chen. Information Visualization: Beyond the Horizon. Springer, second edition, 2006. 
[8] I. Borg and P. J. F. Groenen. Modern Multidimensional Scaling. Springer, second edition, 2005.

[9] K. W. McCain. Mapping authors in intellectual space: a technical overview. J. Am. Soc. Inf. Sci., 41(6):433-443, 1990.

[10] H. D. White and K. W. McCain. Visualizing a discipline: an author co-citation analysis of information science, 1972-1995. J. Am. Soc. Inf. Sci. Tec., 49(4):327-355, 1998.

[11] J. D. Novak and D. B. Gowin. Learning How to Learn. Cambridge University Press, 1984.

[12] H. P. F. Peters and A. F. J. van Raan. Co-word-based science maps of chemical engineering. Part I: representations by direct multidimensional scaling. Research Policy, 22:23-45, 1993.

[13] S. Hinze. Bibliographical cartography of an emerging interdisciplinary discipline: the case of bioelectronics. Scientometrics, 29(3):353-376, 1994.

[14] J. S. Justeson and S. M. Katz. Technical terminology: some linguistic properties and an algorithm for identification of terms in text. Natural Language Engineering, 1:9-27, 1995.

[15] A. Rip and J.-P. Courtial. Co-word maps of biotechnology: an example of cognitive scientometrics. Scientometrics, 6(6):381-400, 1984.

[16] N. J. van Eck and L. Waltman. VOS: a new method for visualizing similarities between objects. In H.-J. Lenz and R. Decker, editors, Advances in Data Analysis: Proc. 30th Ann. Conf. German Classification Society, Studies in Classification, Data Analysis, and Knowledge Organization, pages 299-306. Springer, 2007.

[17] N. J. van Eck, F. Frasincar, and J. van den Berg. Visualizing concept associations using concept density maps. In Proc. 10th Int. Conf. Information Visualisation, pages 270-275, 2006.

[18] D. W. Scott. Multivariate Density Estimation. John Wiley \& Sons, 1992.

[19] J. de Leeuw and J. Meulman. A special jackknife for multidimensional scaling. J. Classif., 3:97-112, 1986. 
[20] W. J. Heiser and J. Meulman. Analyzing rectangular tables by joint and constrained multidimensional scaling. J. of Econometrics, 22:139-167, 1983.

[21] W. J. Heiser and J. Meulman. Constrained multidimensional scaling, including confirmation. Appl. Psych. Meas., 7:381-404, 1983.

[22] S. L. Weinberg, J. D. Carroll, and H. S. Cohen. Confidence regions for INDSCAL using the jackknife and the bootstrap techniques. Psychometrika, 49(4):475-491, 1984.

[23] A. P. Engelbrecht. Computational Intelligence: An Introduction. John Wiley \& Sons, 2003.

[24] A. Konar. Computational Intelligence: Principles, Techniques and Applications. Springer, 2005. 


\section{Publications in the Report Series Research ${ }^{*}$ in Management}

\section{ERIM Research Program: "Business Processes, Logistics and Information Systems"}

2007

India: a Case of Fragile Wireless Service and Technology Adoption?

L-F Pau and J. Motiwalla

ERS-2007-011-LIS

http://hdl.handle.net/1765/9043

Some Comments on the Question Whether Co-occurrence Data Should Be Normalized

Ludo Waltman and Nees Jan van Eck

ERS-2007-017-LIS

http://hdl.handle.net/1765/9401

Extended Producer Responsibility in the Aviation Sector

Marisa P. de Brito, Erwin A. van der Laan and Brijan D. Irion

ERS-2007-025-LIS

Logistics Information and Knowledge Management Issues in Humanitarian Aid Organizations Erwin A. van der Laan, Marisa P. de Brito and S. Vermaesen

ERS-2007-026-LIS

Bibliometric Mapping of the Computational Intelligence Field

Nees Jan van Eck and Ludo Waltman

ERS-2007-027-LIS

Approximating the Randomized Hitting Time Distribution of a Non-stationary Gamma Process

J.B.G. Frenk and R.P. Nicolai

ERS-2007-031-LIS

Application of a General Risk Management Model to Portfolio Optimization Problems with Elliptical Distributed Returns for Risk Neutral and Risk Averse Decision Makers

Bahar Kaynar, S. Ilker Birbil and J.B.G. Frenk

ERS-2007-032-LIS

Optimal Zone Boundaries for Two-class-based Compact 3D AS/RS

Yugang Yu and M.B.M. de Koster

ERS-2007-034-LIS

Portfolios of Exchange Relationships: An Empirical Investigation of an Online Marketplace for IT Services Uladzimir Radkevitch, Eric van Heck and Otto Koppius

ERS-2007-035-LIS

* A complete overview of the ERIM Report Series Research in Management: https://ep.eur.nl/handle/1765/1

ERIM Research Programs:

LIS Business Processes, Logistics and Information Systems

ORG Organizing for Performance

MKT Marketing

F\&A Finance and Accounting

STR Strategy and Entrepreneurship 